Research Paper

\title{
Comparison of the Antialbuminuric Effects of Benidipine and Hydrochlorothiazide in Renin-Angiotensin System (RAS) Inhibitor-Treated Hypertensive Patients with Albuminuria: the COSMO-CKD (COmbination Strategy on Renal Function of Benidipine or Diuretics TreatMent with RAS inhibitors in a Chronic Kidney Disease Hypertensive Population) Study
}

Katsuyuki Ando ${ }^{\circledR}$, Kosaku Nitta ${ }^{2}$, Hiromi Rakugi ${ }^{3}$, Yoshiki Nishizawa ${ }^{4}$, Hitoshi Yokoyama ${ }^{5}$, Takeshi Nakanishi ${ }^{6}$, Naoki Kashihara7, Kimio Tomita ${ }^{8}$, Masaomi Nangaku' ${ }^{9}$, Katsutoshi Takahashi', Masashi Isshiki ${ }^{9}$, Tatsuo Shimosawa ${ }^{10}$, and Toshiro Fujita ${ }^{11}$

1. Division of Molecular Cardiovascular Metabolism, University of Tokyo Graduate School of Medicine, Tokyo, Japan

2. Department of Medicine, Kidney Center, Tokyo Women's Medical University, Tokyo, Japan

3. Department of Geriatric Medicine and Nephrology, Osaka University Graduate School of Medicine, Suita, Japan

4. Metabolism, Endocrinology and Molecular Medicine, Osaka City University Graduate School of Medicine, Osaka, Japan

5. Division of Nephrology, Kanazawa Medical University School of Medicine, Ishikawa, Japan

6. Department of Internal Medicine, Division of Kidney and Dialysis, Hyogo College of Medicine, Nishinomiya, Japan

7. Department of Nephrology and Hypertension, Kawasaki Medical School, Kurashiki, Japan

8. Department of Nephrology, Graduate School of Faculty of Life Science, Kumamoto University, Kumamoto, Japan

9. Department of Nephrology and Endocrinology, University of Tokyo Graduate School of Medicine, Tokyo, Japan

10. Department of Clinical Laboratory, Graduate School of Medicine, University of Tokyo, Tokyo, Japan

11. Division of Clinical Epigenetics, Research Center for Advanced Science and Technology, University of Tokyo, Tokyo, Japan.

$\square$ Corresponding author: Katsuyuki Ando, M.D. Department of Nephrology and Endocrinology, University of Tokyo Graduate School of Medicine, 7-3-1 Hongo, Bunkyo-ku, Tokyo, 113-8655 Japan. Telephone: +81-3-5800-9119, Fax: +81-3-5800-9119 E-mail: katsua-tky@umin.ac.jp

( ) Ivyspring International Publisher. This is an open-access article distributed under the terms of the Creative Commons License (http://creativecommons.org/ licenses / by-nc-nd/3.0/). Reproduction is permitted for personal, noncommercial use, provided that the article is in whole, unmodified, and properly cited.

Received: 2014.03.05; Accepted: 2014.05.14; Published: 2014.06.21

\begin{abstract}
Objective: This study evaluated the non-inferiority of renoprotection afforded by benidipine versus hydrochlorothiazide in hypertensive patients with chronic kidney disease (CKD).

Methods: In this prospective, multicenter, open-labeled, randomized trial, the antialbuminuric effects of benidipine and hydrochlorothiazide were examined in renin-angiotensin system (RAS) inhibitor-treated patients with blood pressure (BP) readings of $\geq 130 / 80 \mathrm{mmHg}$ and $\leq 180 / 110 \mathrm{mmHg}$, a urinary albumin to creatinine ratio (UACR) of $\geq 300 \mathrm{mg} / \mathrm{g}$, and an estimated glomerular filtration rate (eGFR) of $\geq 30 \mathrm{ml} / \mathrm{min} / 1.73 \mathrm{~m}^{2}$. Patients received benidipine ( $\mathrm{n}=176$, final dose: $4.8 \mathrm{mg} /$ day) or hydrochlorothiazide ( $\mathrm{n}=170,8.2 \mathrm{mg} /$ day) for 12 months.

Results: Benidipine and hydrochlorothiazide exerted similar BP- and eGFR-decreasing actions. The UACR values for benidipine and hydrochlorothiazide were 930.8 (95\% confidence interval: 826.I, 1048.7) and 883.I $(781.7,997.7) \mathrm{mg} / \mathrm{g}$ at baseline, respectively. These values were reduced to 790.0 (668.I, 934.2) and $448.5(372.9,539.4) \mathrm{mg} / \mathrm{g}$ at last observation carried forward (LOCF) visits. The non-inferiority of benidipine versus hydrochlorothiazide was not demonstrated (benidipine/hydrochlorothiazide ratio of LOCF value adjusted for baseline: 1.67 (1.40, 1.99)).

Conclusions: The present study failed to demonstrate the non-inferiority of the antialbuminuric effect of benidipine relative to that of hydrochlorothiazide in RAS inhibitor-treated hypertensive patients with macroalbuminuria.
\end{abstract}

Key words: chronic kidney disease, hypertension, renin-angiotensin system inhibitor; L-/N-/T-type calcium channel blocker, thiazide diuretic, urinary albumin. 


\section{Introduction}

Considerable clinical evidence suggests that renin-angiotensin system (RAS) inhibitors (e.g., ramipril and benazepril) are beneficial as first-line agents for the treatment of hypertension in patients with chronic kidney disease (CKD) [1-4]. However, by themselves, RAS inhibitors are unable to maintain an adequate blood pressure (BP) in these individuals. To maintain the $\mathrm{BP}$, second-line depressor agents are therefore required. Accordingly, dihydropyridine-type calcium channel blockers (CCBs) (e.g., felodipine and amlodipine) are frequently used in combination with RAS inhibitors in hypertensive patients with CKD because of their strong BP-lowering properties and minimal adverse effects [5].

However, CCBs are not always able to protect against kidney injury. For example, in the REnoprotection In patients with Nondiabetic chronic renal disease (REIN)-2 trial [6], felodipine did not decrease the incidence of end-stage renal disease in ramipril-treated patients with CKD, despite further lowering the BP. Furthermore, the $\underline{G}$ 으ging Albuminuria Reduction with lotrel in Diabetic patients with hypertension (GUARD) trial [7] showed that the antialbuminuric effect of amlodipine was weaker than that of hydrochlorothiazide, a thiazide diuretic, in benazepril-treated hypertensive patients with type 2 diabetic nephropathy. In addition, urinary protein was not significantly decreased in a meta-analysis of the antiproteinuric effects of dihydropyridine CCBs [8].

On the other hand, certain CCBs, including benidipine (a multifunctional L-type (long-lasting)/Ttype (transient)/N-type (neural) CCB), cilnidipine (a dual L-type/N-type CCB), and azelnidipine (an L-type and sympatholytic CCB), have stronger antialbuminuric effects than others. For example, benidipine decreased urinary protein levels to a greater extent than amlodipine (an L-type CCB) in RAS inhibitor-treated hypertensive patients with stage 3-5 CKD [9]. Benidipine was also more effective than amlodipine in terms of reducing urinary albumin in patients with mild to moderate stage CKD and albuminuria [10]. A more pronounced antiproteinuric effect of benidipine compared to amlodipine has also been reported in hypertensive patients with early-stage CKD [11].

The above studies provide comparisons among CCBs, but not between CCBs and other types of antihypertensives, such as the thiazide diuretics. As noted above, the GUARD trial [7] indicated the inferiority of amlodipine versus hydrochlorothiazide in benazepril-treated hypertensive patients with type 2 diabetic nephropathy. However, there is a paucity of data concerning the non-inferiority of other so-called "renoprotective" CCBs such as benidipine relative to the antihypertensive diuretics. For this reason, we set out to clarify whether benidipine, with demonstrated renoprotective actions in patients with CKD [9-11], could decrease urinary albumin with similar efficacy as hydrochlorothiazide. Hydrochlorothiazide is often used together with an angiotensin receptor blocker (ABR) and/or a thiazide diuretic in CKD patients. Hence, the goal of this study was to examine the non-inferiority of benidipine compared with hydrochlorothiazide when administered to RAS inhibitor-treated hypertensive patients with CKD and macroalbuminuria.

\section{Methods}

A prospective, multicenter, open-labeled, randomized trial, the COmbination Strategy on renal function of benidipine or diuretics treatMent with RAS inhibitOrs in Chronic Kidney Disease hypertensive population (COSMO-CKD) trial, was performed in clinics and hospitals in Japan from July 2009 to March 2013. The trial was registered with the University Hospital Medical Information Network-Clinical Trials Registry (UMIN-CTR) under trial identification number UMIN000002143. The trial was approved by the Institutional Review Board of the University of Tokyo Clinical Research Center (reference number P2008042-11X) and by the review boards of all other participating medical facilities. The trial was conducted in accordance with the principles of the Declaration of Helsinki. Written informed consent was obtained from all participating patients after they received oral and written explanations about the study protocol.

\section{Participants}

Hypertensive patients with albuminuria under treatment with an RAS inhibitor (ARB or angiotensin-converting enzyme (ACE) inhibitor) were recruited for this study. The inclusion criteria were: 1) outpatient systolic and diastolic BP readings of $\geq$ $130 / 80 \mathrm{mmHg}$; 2) a pretreatment urinary albumin to creatinine ratio (UACR) (the average of two measured values) of $\geq 300 \mathrm{mg} / \mathrm{g}$; 3) an estimated glomerular filtration rate (eGFR) [12] of $\geq 30 \mathrm{ml} / \mathrm{min} / 1.73 \mathrm{~m}^{2} ; 4$ ) between 20 and 80 years of age; 5) a duration of antihypertensive treatment with RAS inhibitors of $\geq 3$ months prior to enrollment; and 6) no treatment with CCBs or diuretics of any kind for at least 3 months prior to enrollment. The exclusion criteria were: 1) outpatient systolic and diastolic BP readings of $\geq$ $180 / 110 \mathrm{mmHg}$; 2) a hypertensive emergency requiring intravenous administration of any antihypertensive agent; 3) administration of an adrenocorticosteroid or an immunosuppressant, or long-term $(\geq 2$ 


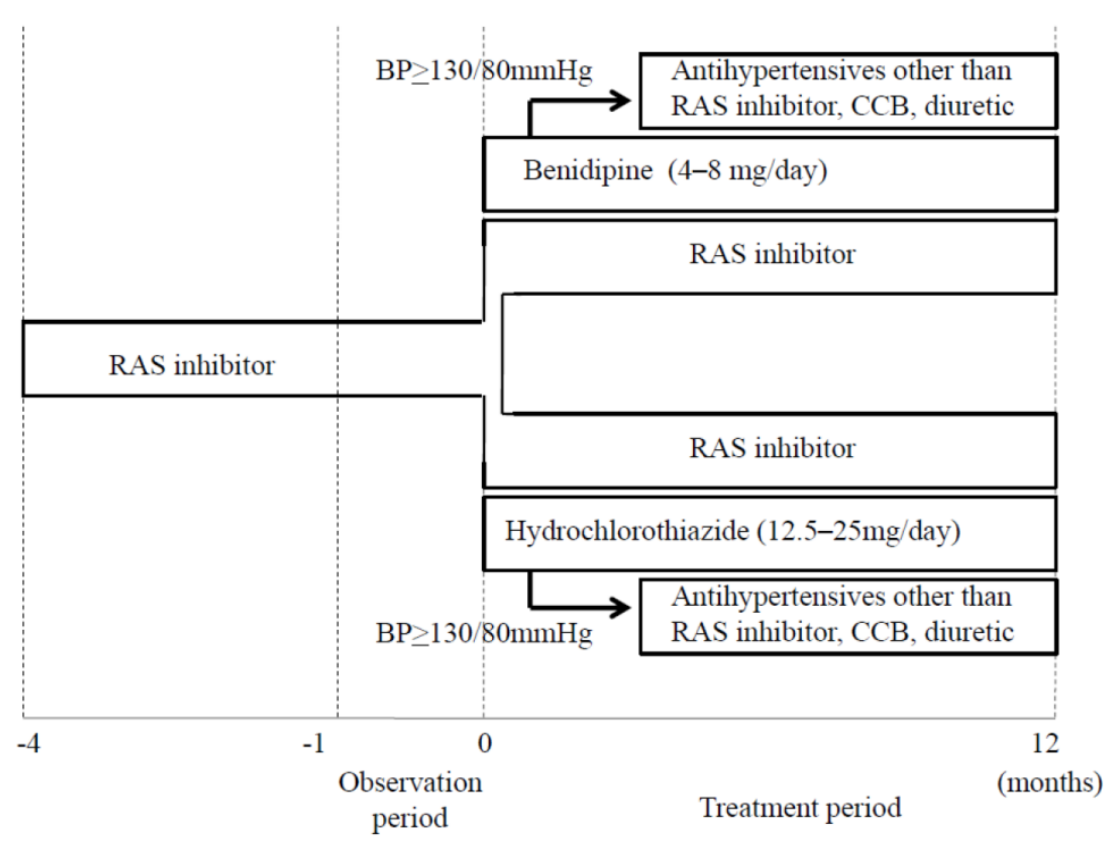

Figure I. Study protocol. BP, blood pressure; CCB, calcium channel blocker; RAS, renin-angiotensin system.

inhibitor was not changed during the treatment period.

The following factors were used for stratified randomization: 1) systolic $\mathrm{BP}(<140 \mathrm{mmHg}$, $\geq 140 \mathrm{mmHg}$ ) and 2) UACR $(<1000 \mathrm{mg} / \mathrm{g}, \geq 1000$ $\mathrm{mg} / \mathrm{g})$. The target BP was $<130 / 80$ $\mathrm{mmHg}$. If benidipine or hydrochlorothiazide plus the RAS inhibitor failed to reduce BP to the target level, additional antihypertensive drugs of a different type (i.e., not a $\mathrm{CCB}$, diuretic, or RAS inhibitor) were administered. The treatment period in each case was 12 months.

\section{Outcome measures}

The primary endpoint was the change in the UACR $(\mathrm{mg} / \mathrm{g})$ from the baseline to the endpoint (endpoint/baseline ratio). The UACR at endpoint was determined in spot

weeks) administration of nonsteroidal anti-inflammatory drugs (NSAIDs); 4) a past history of severe adverse reaction to CCBs, thiazide diuretics, ARBs, or ACE inhibitors; 5) type 1 diabetes or type 2 diabetes requiring hospitalization due to high hemoglobin A1c content ( $\geq 9.0 \%$ ), extremely high blood glucose, or diabetic ketoacidosis; 6) cerebrovascular disease occurring within 6 months of enrollment; 7) severe heart failure (New York Heart Association (NYHA) class $\geq$ III), severe arrhythmia (frequent ventricular or atrial extrasystole, prolonged ventricular tachycardia, atrial tachyarrhythmia with severe tachycardia, atrial fibrillation or flutter with severe tachycardia, sick sinus syndrome with severe bradycardia, or atrio-ventricular block with severe bradycardia), angina, or myocardial infarction within 6 months of enrollment; 8) aspartate aminotransferase (AST) or alanine aminotransferase (ALT) levels of $>5$ times the upper limit; and 9) pregnancy, the possibility of pregnancy, or a desire to become pregnant.

\section{Interventions}

After confirming patient eligibility during the observation period (Figure 1), each individual was randomly allocated to one of two groups, the benidipine group or the hydrochlorothiazide group. Benidipine was initiated at $4 \mathrm{mg} /$ day, followed by adjustment to 4-8 mg/day, while hydrochlorothiazide was initiated at $6.25 \mathrm{mg} /$ day, followed by adjustment to $6.25-12.5 \mathrm{mg} /$ day. Each drug was given in combination with one or more RAS inhibitors (an ARB and/or an ACE inhibitor) during the treatment period (Figure 1), and the dose of the ARB or the ACE urine samples after 12 months of benidipine or hydrochlorothiazide treatment, and the UACR at baseline (the average of two consecutive measurements) was determined during a 4-week pretreatment period. The resulting endpoint/baseline ratios were then compared between the benidipine and hydrochlorothiazide arms. Laboratory tests were performed at a central laboratory (Mitsubishi Chemical Medicine Inc., Tokyo, Japan). The urinary albumin level was measured by using the Bromcresol green photometric method (IatroFine ALB II), and the urinary creatinine level was measured by using an enzymatic colorimetric assay (IatroLQ CRE(A) II).

Secondary outcomes included the absolute values of the UACR at each time point; the eGFR, calculated according to the modified "Modification of Diet in Renal Disease (MDRD)" formula set forth by the Japanese Society of Nephrology (male: eGFR $\left(\mathrm{ml} / \mathrm{min} / 1.73 \mathrm{~m}^{2}\right)=194 \times$ age $^{-0.287} \times$ serum creatinine-1.094; female: same as for male, with further multiplication by a factor of 0.739) [12]; the CKD stage [13]; urinary liver-type free fatty acid-binding protein (L-FABP) levels; serum creatinine and blood urea nitrogen (BUN) content; BP readings; renal events (initiation of dialysis or renal transplantation); cerebro-cardiovascular events (cerebro-cardiovascular death (fatal myocardial infarction, fatal heart failure, sudden death, fatal stroke, or death due to other cardiovascular causes) or hospitalization due to a cerebro-cardiovascular disease (nonfatal myocardial infarction, angina, heart failure, cerebral bleeding, cerebral infarction, or transient cerebral ischemic attack)); and other adverse events. 


\section{Statistical Analysis}

All analyses were performed using SAS version 9.1 (SAS Institute, Cary, NC, USA). The safety of the treatment assignments was assessed in a safety analysis set. The efficacy of the treatment assignments were analyzed in the full analysis set. Subjects who did not meet the eligibility criteria, who were not administered the assigned drugs (benidipine or hydrochlorothiazide), or who had no relevant data after randomization were excluded from the full analysis. All data are given as the mean \pm standard deviation (SD) or the mean together with the $95 \%$ confidence interval.

For the analysis of the non-inferiority of the antialbuminuric effect of benidipine versus hydrochlorothiazide when prescribed in combination with RAS inhibitors, the value of the endpoint/baseline ratio of the UACR in the benidipine group was divided by that of the UACR in the hydrochlorothiazide group. An inferiority margin was assumed as $\geq 0.80$ or $\leq 1.25$. One-hundred-and-seventy patients were required in each treatment group for a power of $80 \%$ with a significance level of $2.5 \%$ (one-sided test) to detect the non-inferiority of benidipine when the SD of the natural logarithm of the UACR was assumed to be 0.73 . With the added expectation of $15 \%$ of the patients withdrawing from the study, a plan was formulated to recruit approximately 200 patients to each group. In actuality, 585 patients were recruited, and 365 were randomized.

\section{Results}

As noted above, 585 patients were recruited into the COSMO-CKD trial. Of these 585 individuals, 365 were randomized. Nineteen individuals were not administered an allocated drug and were therefore eliminated from the trial. Data corresponding to the remaining 346 patients (benidipine, $\mathrm{n}=176$; hydrochlorothiazide, $n=170$ ) were used for the analysis of drug safety. However, only 344 of these individuals (benidipine, $\mathrm{n}=175$; hydrochlorothiazide, $\mathrm{n}=169$ ) were included in the analysis of efficacy (Table 1), because two patients (benidipine group, $\mathrm{n}=1$; hydrochlorothiazide group, $\mathrm{n}=1$ ) did not meet the eligibility criteria. Furthermore, only 277 of 346 subjects (benidipine, $\mathrm{n}=143$; hydrochlorothiazide, $\mathrm{n}=134$ ) completed the study regimen. The patient characteristics at baseline $(n=344)$ are shown in Table 1 . The baseline data were almost identical between the two groups.

Baseline UACR was 930.8 (95\% confidence interval, 826.1, 1048.7) $\mathrm{mg} / \mathrm{g}$ in the benidipine group and $883.1(781.7,997.7) \mathrm{mg} / \mathrm{g}$ in the hydrochlorothiazide group (Figure 2a). After 12 months of drug treatment, the UACR values were reduced to 783.1 for benidipine and $383.6 \mathrm{mg} / \mathrm{g}$ for hydrochlorothiazide. The last observation carried forward (LOCF) value of the UACR was $790.0(668.1,934.2) \mathrm{mg} / \mathrm{g}$ for benidipine and $448.5(372.9,539.4) \mathrm{mg} / \mathrm{g}$ for hydrochlorothiazide, resulting in endpoint/baseline UACR ratios of $0.85(0.76,0.95)$ (benidipine group) and 0.51 $(0.44,0.58)$ (hydrochlorothiazide group). The ratio (benidipine/hydrochlorothiazide) of the endpoint/baseline data sets was $1.67(1.40,1.99)$. Thus, the non-inferiority of benidipine versus hydrochlorothiazide was not demonstrated (Figure $\mathbf{2 b}$ ).

Table I. Patient Characteristics.

\begin{tabular}{|c|c|c|c|}
\hline & Benidipine & $\begin{array}{l}\text { Hydrochloro- } \\
\text { thiazide }\end{array}$ & P value \\
\hline $\mathrm{n}$ & 175 & 169 & \\
\hline Male/female & $124 / 51$ & $111 / 58$ & $0.354^{*}$ \\
\hline Age (years) & $59.5 \pm 11.5$ & $58.4 \pm 12.1$ & $0.380^{* *}$ \\
\hline BMI $\left(\mathrm{kg} / \mathrm{m}^{2}\right)$ & $26.07 \pm 5.44$ & $25.83 \pm 4.48$ & $0.654^{* *}$ \\
\hline Systolic BP (mmHg) & $144.4 \pm 12.5$ & $143.7 \pm 12.5$ & $0.641^{* *}$ \\
\hline Diastolic BP (mmHg) & $83.6 \pm 8.9$ & $84.5 \pm 9.0$ & $0.332^{* *}$ \\
\hline Pulse rate (bpm) & $75.8 \pm 10.8$ & $76.5 \pm 10.7$ & $0.513^{* *}$ \\
\hline $\begin{array}{l}\text { Serum total cholesterol } \\
(\mathrm{mg} / \mathrm{dl})\end{array}$ & $200.4 \pm 35.3$ & $200.8 \pm 36.9$ & $0.919^{* *}$ \\
\hline $\begin{array}{l}\text { Serum LDL cholesterol } \\
(\mathrm{mg} / \mathrm{dl})\end{array}$ & $117.8 \pm 35.4$ & $114.7 \pm 32.2$ & $0.403^{* *}$ \\
\hline $\begin{array}{l}\text { Serum HDL cholesterol } \\
(\mathrm{mg} / \mathrm{dl})\end{array}$ & $56.8 \pm 15.9$ & $55.3 \pm 15.1$ & $0.371^{* *}$ \\
\hline Serum triglycerides $(\mathrm{mg} / \mathrm{dl})$ & $182.4 \pm 143.4$ & $193.1 \pm 145.0$ & $0.493^{* *}$ \\
\hline Blood sugar (mg/dl) & $141.5 \pm 53.0$ & $140.5 \pm 54.5$ & $0.864^{* *}$ \\
\hline Hemoglobin A1c (\%) & $6.37 \pm 1.16$ & $6.22 \pm 1.12$ & $0.226^{* *}$ \\
\hline AST (IU/1) & $25.7 \pm 14.1$ & $24.8 \pm 10.9$ & $0.510^{* *}$ \\
\hline $\operatorname{ALT}(\mathrm{IU} / 1)$ & $25.4 \pm 17.5$ & $25.5 \pm 21.2$ & $0.962^{* *}$ \\
\hline$\gamma-\mathrm{GTP}(\mathrm{IU} / 1)$ & $39.7 \pm 33.7$ & $47.8 \pm 52.3$ & $0.088^{* *}$ \\
\hline Serum sodium (mEq/l) & $140.5 \pm 2.7$ & $140.2 \pm 2.5$ & $0.286^{* *}$ \\
\hline Serum potassium $(\mathrm{mEq} / \mathrm{l})$ & $4.36 \pm 0.40$ & $4.36 \pm 0.48$ & $1.000^{* *}$ \\
\hline \multicolumn{4}{|l|}{ Antihypertensive agents } \\
\hline $\mathrm{ARB}$ & 161 & 159 & \\
\hline ACE inhibitor & 27 & 23 & \\
\hline$\alpha$ blocker & 3 & 7 & \\
\hline$\beta$ blocker & 5 & 6 & \\
\hline Others & 6 & 4 & \\
\hline \multicolumn{4}{|l|}{ Renal disease } \\
\hline Diabetic nephropathy & 99 & 78 & \\
\hline Glomeruronephritis & 40 & 56 & \\
\hline Others & 35 & 37 & \\
\hline \multicolumn{4}{|l|}{ Complications } \\
\hline Dyslipidemia & 100 & 79 & \\
\hline Diabetes & 112 & 88 & \\
\hline Liver dysfunction & 16 & 10 & \\
\hline Cerebrovascular disease & 3 & 6 & \\
\hline Myocardial infarction & 0 & 0 & \\
\hline Angina pectoris & 2 & 2 & \\
\hline Heart failure & 0 & 1 & \\
\hline
\end{tabular}

$\mathrm{ACE}$, angiotensin-converting enzyme; ARB, angiotensin receptor blocker; ALT, alanine aminotransferase; AST, aspartate aminotransferase; BMI, body mass index; $\mathrm{BP}$, blood pressure; $\gamma$-GTP, gamma-glutamyl transpeptidase; HDL, high density lipoprotein; LDL, low density lipoprotein.

${ }^{*}$ Fisher's exact test, ${ }^{* *} t$-test. 

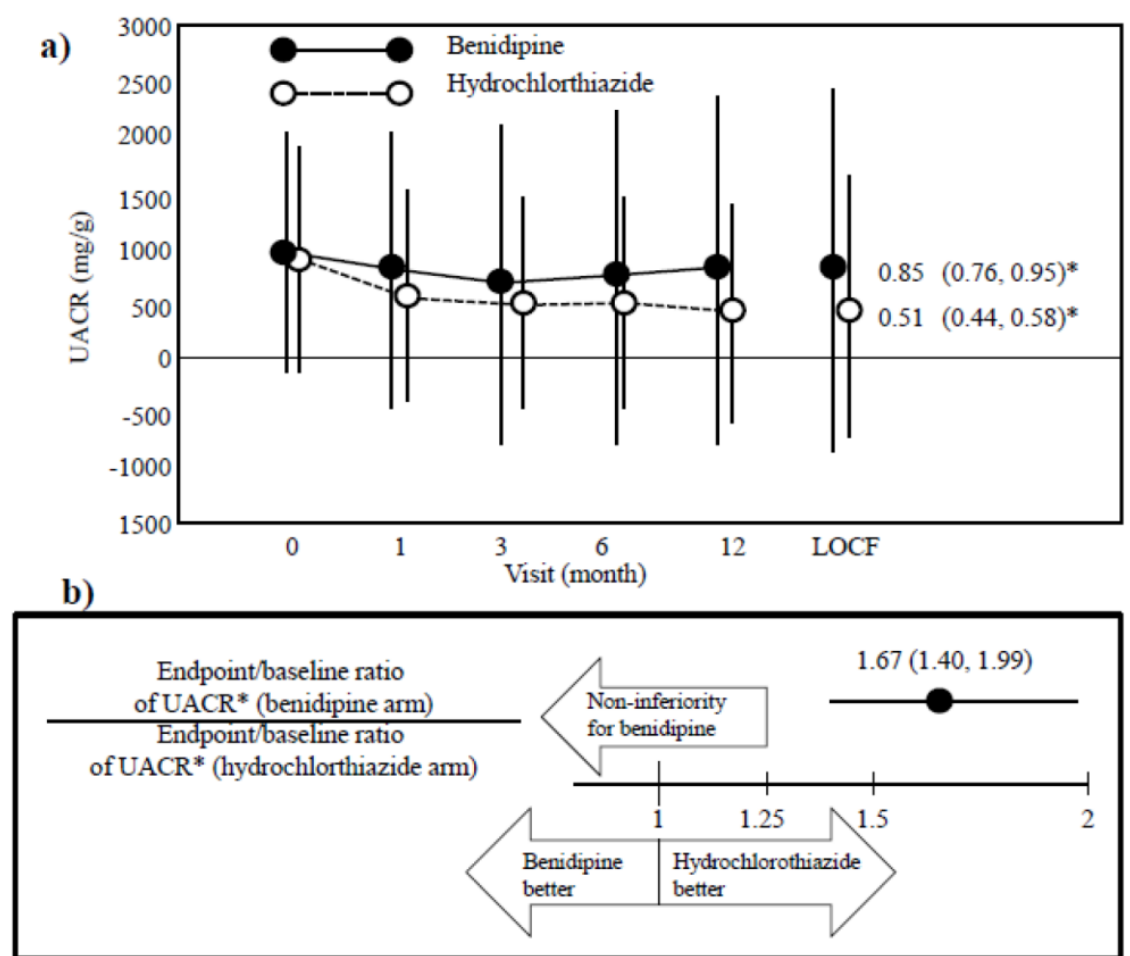

Figure 2. Changes in the urinary albumin/creatinine ratio (UACR). a) The decrease in UACR was greater in the hydrochlorothiazide group than in the benidipine group, as indicated by the endpoint/baseline ratio of the UACR for each drug. Data (black and white circles) are given as the mean \pm the standard deviation (SD). LOCF, last observation carried forward. * The endpoint/baseline ratio of the UACR for each drug is given as the mean ( $95 \%$ confidence interval). b) The endpoint/baseline data are given as the ratio of the benidipine arm/hydrochlorothiazide arm (mean and $95 \%$ confidence interval). The non-inferiority of benidipine was not demonstrated.

Serum creatinine levels were similar at baseline (0.961 $(0.919,1.005) \mathrm{mg} / \mathrm{dl}$, benidipine; 0.967 (0.922, $1.014) \mathrm{mg} / \mathrm{dl}$, hydrochlorothiazide) and at the LOCF (1.039 $(0.986,1.096) \mathrm{mg} / \mathrm{dl}$, benidipine; 1.054 (0.998, $1.114) \mathrm{mg} / \mathrm{dl}$, hydrochlorothiazide). However, serum creatinine levels were slightly and similarly increased by both treatments (Figure 3a). By contrast, eGFR values were also similar at baseline (57.91 (55.31, $60.64) \mathrm{ml} / \mathrm{min} / 1.73 \mathrm{~m}^{2}$, benidipine; $57.01(54.05,54.05)$ $\mathrm{ml} / \mathrm{min} / 1.73 \mathrm{~m}^{2}$, hydrochlorothiazide) and at the LOCF $\left(52.81(49.94,55.84) \mathrm{ml} / \mathrm{min} / 1.73 \mathrm{~m}^{2}\right.$, benidipine; $51.55(43.36,54.95) \mathrm{ml} / \mathrm{min} / 1.73 \mathrm{~m}^{2}$, hydrochlorothiazide) (Figure $3 \mathbf{b}$ ), but the eGFR values were slightly and similarly decreased.

Table 2 shows that the distribution of CKD stages, as assessed by the eGFR, was largely comparable between baseline and the LOCF for both drug groups. CKD staging was also similar between the two groups at each time point. However, a marked increase (from $1.2(0.1,4.2) \%$ at baseline to 10.1 (6.0, $15.7) \%$ at the LOCF) was observed in the number of stage 4 patients after hydrochlorothiazide treatment. At the same time point, the number of stage 4 patients in the benidipine group increased from $1.2(0.1,4.2) \%$ at the baseline to $5.3(2.4,9.8) \%$ at the LOCF.
Table 2. CKD stages due to the eGFR at baseline and at the LOCF.

\begin{tabular}{|c|c|c|c|c|c|c|c|}
\hline \multirow{3}{*}{$\mathrm{N}$} & \multicolumn{3}{|c|}{ Benidipine } & \multicolumn{4}{|c|}{ Hydrochlorothiazide } \\
\hline & \multicolumn{3}{|c|}{171} & \multicolumn{3}{|c|}{168} & \multirow[t]{2}{*}{ P value } \\
\hline & $\mathrm{N}$ & $\%$ & $95 \% \mathrm{CI}$ & $\mathrm{n}$ & $\%$ & $95 \% \mathrm{CI}$ & \\
\hline \multicolumn{8}{|c|}{ Baseline } \\
\hline Stage 1 & 9 & 5.3 & $2.4,9.8$ & 16 & 9.5 & $5.5,15.0$ & 0.150 \\
\hline Stage 2 & 78 & 45.6 & $38.0,53.4$ & 61 & 36.3 & $29.0,44.1$ & 0.098 \\
\hline Stage 3 & 82 & 48.0 & $40.3,55.7$ & 89 & 53.0 & $45.1,60.7$ & 0.386 \\
\hline Stage 4 & 2 & 1.2 & $0.1,4.2$ & 2 & 1.2 & $0.1,4.2$ & 1.000 \\
\hline Stage 5 & 0 & 0.0 & $0.0,2.1$ & 0 & 0.0 & $0.0,2.2$ & - \\
\hline \multicolumn{8}{|l|}{ LOCF } \\
\hline Stage 1 & 7 & 4.1 & $1.7,8.3$ & 12 & 7.1 & $3.7,12.1$ & 0.246 \\
\hline Stage 2 & 66 & 38.6 & $31.3,46.3$ & 50 & 29.8 & $23.0,37.3$ & 0.109 \\
\hline Stage 3 & 88 & 51.5 & $43.7,59.2$ & 88 & 52.4 & $44.5,60.1$ & 0.914 \\
\hline Stage 4 & 9 & 5.3 & $2.4,9.8$ & 17 & 10.1 & $6.0,15.7$ & 0.105 \\
\hline Stage 5 & 1 & 0.6 & $0.0,3.2$ & 1 & 0.6 & $0.0,3.3$ & 1.000 \\
\hline
\end{tabular}

CI, confidence interval; CKD, chronic kidney disease; eGFR, estimated glomerular filtration rate; LOCF, last observation carried forward.

* Fisher's exact test.

BUN values were similar between the two drugs at baseline $(17.57(16.85,18.32) \mathrm{mg} / \mathrm{dl}$, benidipine; $17.28(16.49,18.11) \mathrm{mg} / \mathrm{dl}$, hydrochlorothiazide) and at the LOCF $(18.14(17.31,19.01) \mathrm{mg} / \mathrm{dl}$, benidipine; $18.84(17.84,19.89) \mathrm{mg} / \mathrm{dl}$, hydrochlorothiazide). However, although BUN levels did not appreciably change over the course of the study in the benidipine group (endpoint/ baseline ratio, $1.02(0.98,1.07))$, they were slightly albeit significantly increased in the hy- 
drochlorothiazide group $(1.09(1.05,1.13))$. Hence, the non-inferiority of benidipine was demonstrated in terms of BUN content (benidipine arm/hydrochlorothiazide arm, $0.98(0.89,1.00))$.

Urinary L-FABP values were also comparable between the two drug groups at baseline (15.20 (12.92, $17.88) \mu \mathrm{g} / \mathrm{g}$, benidipine; $13.94(11.70,16.60) \mu \mathrm{g} / \mathrm{g}$, hydrochlorothiazide) and at the LOCF (18.67 (15.47, 22.53) $\mu \mathrm{g} / \mathrm{g}$, benidipine; $13.44(11.22,16.11) \mu \mathrm{g} / \mathrm{g}$, hydrochlorothiazide). L-FABP levels showed a slight but significant increase in the benidipine group (endpoint/baseline ration, $1.27(1.10,1.46))$, but not in the hydrochlorothiazide group (endpoint/baseline ratio, $0.98(0.84,1.13))$. The non-inferiority of benidipine was not suggested (benidipine arm/hydrochlorothiazide arm, $1.30(1.06,1.59))$.

Systolic and diastolic BP readings showed similar baseline values between the two drug groups. The systolic BP was $144.4(142.5,146.2) \mathrm{mmHg}$ for benidipine and $143.7(141.9,145.6) \mathrm{mmHg}$ for hydrochlorothiazide, and the diastolic BP was $83.6(82.2,84.9)$ $\mathrm{mmHg}$ for benidipine and $84.5(83.1,85.9) \mathrm{mmHg}$ for hydrochlorothiazide, respectively. Systolic and diastolic BP both decreased with drug treatment, and their decrements were similar between the two groups (Figure 4).

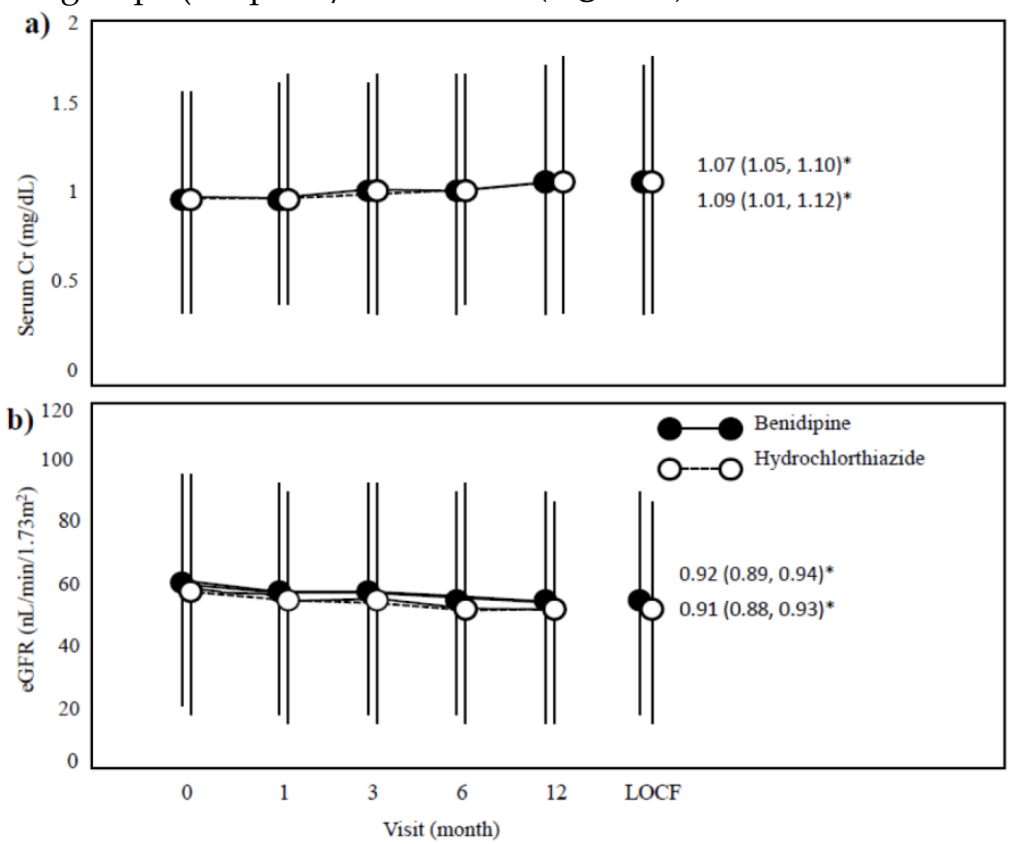

Figure 3. Changes in serum creatinine (a) and estimated glomerular filtration rate (eGFR) (b). Data (black and white circles) are given as the mean \pm the standard deviation (SD). LOCF, last observation carried forward. * The endpoint/baseline ratios for benidipine and hydrochlorothiazide are each given as the mean ( $95 \%$ confidence interval).
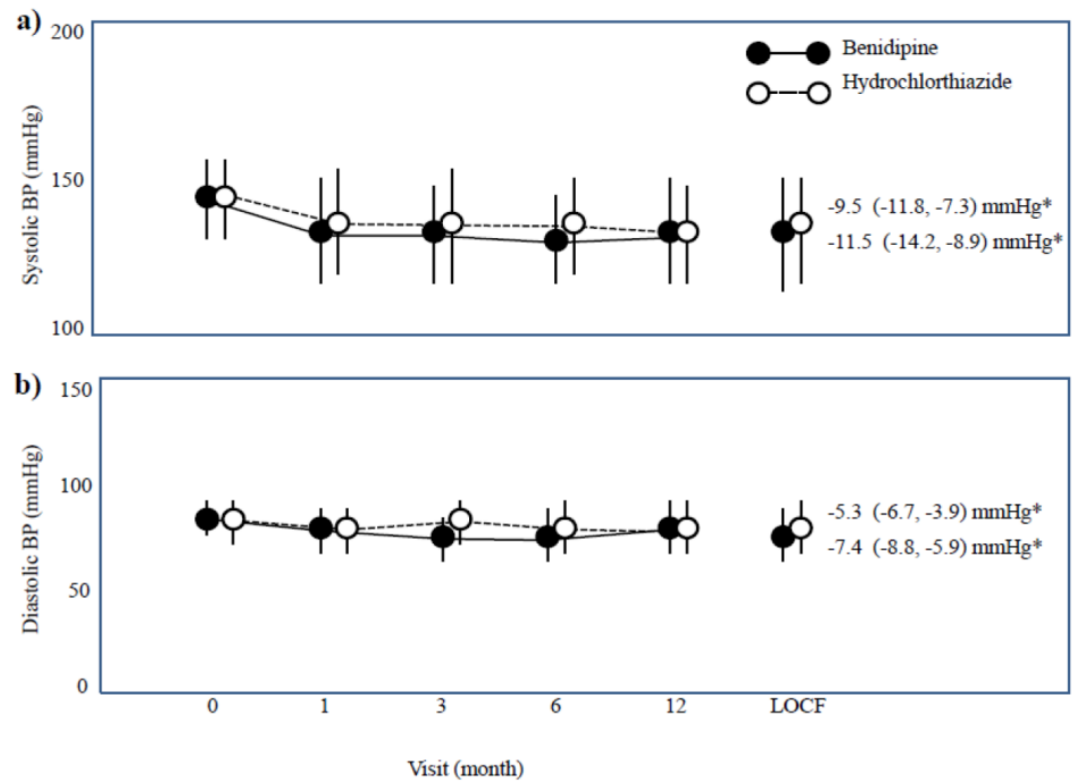

Figure 4. Changes in systolic and diastolic blood pressure (BP). Data are given as the mean \pm the standard deviation (SD). LOCF, last observation carried forward. * Changes in the BP from the baseline to the endpoint are shown as the mean ( $95 \%$ confidence interval). 
Adverse events occurred in 65 of 176 patients $(36.9 \%)$ in the benidipine group and 74 of 170 patients $(43.5 \%)$ in the hydrochlorothiazide group. Severe adverse events included one case of vitreous hemorrhage with aggravation of diabetic retinopathy and one case of cerebral bleeding in the benidipine group, and one case each of arteriosclerotic obliteration, gangrene of right third toe, and cerebral infarction with death in the hydrochlorothiazide group. There were no patients in whom renal events occurred, serious or otherwise.

\section{Discussion}

The present study failed to demonstrate the non-inferiority of the antialbuminuric effect of benidipine versus hydrochlorothiazide in RAS inhibitor-treated hypertensive patients with macroalbuminuria. The effect of benidipine appeared to be rather inferior to that of hydrochlorothiazide in terms of UACR (Figure $2 b$ ), even though the previous studies report that benidipine decreases urinary albu$\mathrm{min} /$ protein more efficaciously in patients with a wide range of CKD stages than other CCBs, such as amlodipine [9-11]. Therefore, although we did not directly compare antialbuminuric actions between benidipine and amlodipine, the current investigation together with the previous data [9-11] suggest that the potency of benidipine to decrease urinary albumin might be intermediate between the less effective CCBs and the more effective thiazide diuretics.

Benidipine and hydrochlorothiazide decreased the eGFR to a similar extent in the present COSMO-CKD trial, although its actions of both drugs on the kidney were fairly weak. On the other hand, amlodipine had a slightly but significantly lower propensity than hydrochlorothiazide to decrease the eGFR in the GUARD study [7]. These results indirectly suggest that amlodipine, but not benidipine, exclusively dilates the afferent artery via L-type calcium channel blockade to increase glomerular pressure, as shown previously [14]. Hence the blockade of $\mathrm{T}$ - and $\mathrm{N}$-type calcium channels by benidipine, aside from its L-type calcium channel-blocking effects, may dilate both afferent and efferent arteries, leading to decreasing glomerular pressure, which might protect kidney. However, weaker antialbuminuric effect of benidipine than hydrochlorothiazide suggest that benidipine is less beneficial on the kidney, although both drugs did similarly reduce office systolic and diastolic BP.

Thus, thiazide diuretics may have a potent effect to decrease urinary albumin. Recently, the combination of losartan and hydrochlorothiazide decreased morning BP to a greater extent than high-dose losartan. The combination was also associated with a larger decrease in the UACR [15]. Furthermore, the effects of the thiazide diuretic to ameliorate circadian BP (from a non-dipper pattern to a dipper pattern) and to suppress proteinuria have been demonstrated in RAS inhibitor-treated patients with immunoglobulin A nephropathy [16]. Daytime salt retention is proposed to cancel the normal nighttime reduction in the BP; nighttime high $\mathrm{BP}$ accelerates pressure natriuresis to excrete sodium retained during the day. However, diuretics are thought to attenuate daytime sodium retention, resulting in reduced nighttime BP. Nonetheless, daytime and nighttime BP were not examined in the present study. At any rate, thiazide diuretics, which have a different mechanism of action from RAS inhibitors to decrease urinary albumin, may confer greater renoprotective in combination with RAS inhibitors than L-/T-/N-type CCBs such as benidipine, which has a similar renoprotective mechanism (efferent arteriole vasodilation) as RAS inhibitors.

Another recent study [17] demonstrated the non-inferiority of the antialbuminuric effect of azelnidipine, another so-called "renoprotective" CCB [18], compared with the thiazide diuretic, trichloromethiazide. Because several different mechanisms have been attributed to the "renoprotective" CCBs (e.g., T-type calcium channel blockade (benidipine), N-type calcium channel blockade (benidipine and cilnidipine), and sympatholytic effects (azelnidipine)), the extent of antialbuminuric action might be different among these agents.

In addition, the subjects of the present study and the above-mentioned study [17] showed different degrees of albuminuria; $\geq 300 \mathrm{mg} / \mathrm{g}$ and $30-600$ $\mathrm{mg} / \mathrm{g}$, respectively. Recently, Ogawa et al. demonstrated that the antialbuminuric effects of ARB were weakened in patients with $\geq 1,000 \mathrm{mg} / \mathrm{g}$ of UACR [19]. Thus, amelioration of glomerular microcirculation may not effectively decrease urinary albumin in CKD patients with the advanced renal dysfunction. Therefore, the non-inferiority of benidipine versus hydrochlorothiazide might be observed in RAS inhibitor-treated CKD patients with lower UACR values.

Another consideration is that the majority of the patients included in the present study had diabetes (Table 1). Macroalbuminuric patients with diabetes may have advanced diabetic vascular damage, which might also suppress the effectiveness of other "renoprotective" CCB [20]. In addition, there are few reports that compare the antialbuminuric effects between so-called "renoprotective" CCB and thiazide diuretics. Moreover, future investigations are required to examine whether the antiproteinuric effect of benidipine and other "renoprotective" CCBs diuretic is non-inferior compared with thiazides in pa- 
tients with microalbuminuria.

The present study has several limitations. First, UACR values in spot urine samples can vary with each measurement, even in the same patient with the same drug treatment. In this regard, measurement of urinary albumin excretion in samples collected over a 24-h period or repeated measurements of the first morning void sample may be more accurate than a spot sample. Second, sample size estimation was done in the absence of previous reports comparing the antialbuminuric effects of "renoprotective" CCBs and thiazide diuretics. For this reason, we cannot guarantee that the sample size was sufficient.

In conclusion, the COSMO-CKD trial failed to demonstrate the non-inferiority of the antialbuminuric effect of benidipine relative to hydrochlorothiazide. Several previous studies showed the renoprotective actions of benidipine in CKD patients with various stages of disease, as well as its potentially beneficial actions on glomerular microcirculation. Thus, the renoprotective effect of benidipine might be limited compared with more potent thiazide diuretics, but more pronounced than that of other CCBs. Further studies are required to clarify in the subset of CKD patients in which benidipine most effectively decreases urinary albumin content.

\section{Acknowledgments}

The COSMO-CKD trial was funded by the Kidney Foundation of Japan.

\section{Competing Interests}

The first author (K.A.) is endowed by Daiichi-Sankyo, Co., Ltd. (Tokyo, Japan). The last author (T.F.) is endowed by Mochida Pharmaceutical Co., Ltd., Chugai Pharmaceutical Co., Ltd., Mitsubishi Tanabe Pharma Co., Ltd., Kyowa Hakko Kirin Co., Ltd., MSD Co., Ltd., Toray Industries, Inc., and Terumo Cooperation (all in Tokyo, Japan).

\section{References}

1. Ando K, Fujita T. Anti-diabetic effect of blockade of the renin-angiotensin system. Diab Obes Metab 2006;8:396-403.

2. Shimamoto K, Ando K, Fujita T, et al. The Japanese Society of Hypertension Committee for Guidelines for the Management of Hypertension: The Japanese Society of Hypertension Guidelines for the Management of Hypertension (JSH 2014). Hypertens Res 2014;37:253-392.

3. TheTask Force for the management ofarterial hypertension of the European Society ofHypertension (ESH) and of the European Society of Cardiology (ESC). 2013 ESH/ESC Guidelines for the management of arterial hypertension. J Hypertens. 2013,31:1281-1357

4. Chobanian AV, Bakris GL, Black HR, et al. Joint National Committee on Prevention, Detection, Evaluation, and Treatment of High Blood Pressure: National Heart, Lung, and Blood Institute; National High Blood Pressure Education Program Coordinating Committee. Seventh report of the Joint National Committee on Prevention, Detection, Evaluation, and Treatment of High Blood Pressure. Hypertension 2003;42:1206-1252.

5. Kloke HJ, Branten AJ, Huysmans FT, et al. Antihypertensive treatment of patients with proteinuric renal diseases: risks or benefits of calcium channel blockers? Kidney Int 1998;53:1559-1573.
6. Roggenenti P, Perna A, Loriga G, et al. REIN-2 study group: Blood-pressure control for renoprotection in patients with non-diabetic chronic renal disease (REIN-2): multicentre, randomised controlled trail. Lancet 2005;365:939-946.

7. Bakris GL, Toto RD, McCullough PA, et al. GUARD (gauging albuminuria reduction with Lotrel in diabetic patients with hypertension) study investigators: Effects of different ACE inhibitor combinations on albuminuria: results of the GUARD study. Kidney Int. 2008;73:1303-1309.

8. Bakris GL, Weir M, Secic M, et al. Differential effects of calcium antagonist subclasses on markers of nephropathy progression. Kidney Int. 2004;65:1991-2002.

9. Abe M, Okada K, Maruyama T, et al. Comparison of the antiproteinuric effects of the calcium channel blockers benidipine and amlodipine administered in combination with angiotensin receptor blockers to hypertensive patients with stage 3-5 chronic kidney disease. Hypertens Res. 2009;32:270-275.

10. Abe M, Okada K, Maruyama N, et al. Benidipine reduces albuminuria and plasma aldosterone in mild-to-moderate stage chronic kidney disease with albuminuria. Hypertens Res. 2011;34:268-273.

11. Nakamura T, Sato E, Fujiwara N, et al. Comparative effects of benidipine and amlodipine on proteinuria, urinary $8-\mathrm{OHdG}$, urinary L-FABP, and inflammatory and atherosclerosis markers in early-stage chronic kidney disease. $\mathrm{Am}$ J Med Sci. 2010;339:157-163.

12. Matsuo S, Imai E, Horio $\mathrm{M}$, et al. Collaborators developing the Japanese equation for estimated GFR. Revised equations for estimated GFR from serum creatinine in Japan. Am J Kidney Dis 2009;53:982-992.

13. Japanese Society of Nephrology. Evidence-based Clinical Practice Guidebook for CKD. Jpn J Nephrol. 2013;55:585-860. (in Japanese).

14. Hayashi K, Wakino S, Sugano N, et al. $\mathrm{Ca}^{2+}$ channel subtypes and pharmacology in the kidney. Circ Res 2007;100:342-353.

15. Ueda T, Kai H, Imaizumi T. On behalf of the MAPPY Study Investigators. Losartan/hydrochlorothiazide combination vs. high-dose losartan in patients with morning hypertension-a prospective, randomized, open-labeled, parallel-group, multicenter trial. Hypertens Res 2012; 35:708-714.

16. Uzu T, Harada T, Namba $T$, et al. Thiazide diuretics enhance nocturnal blood pressure fall and reduce proteinuria in immunoglobulin A nephropathy treated with angiotensin II modulators. J Hypertens. 2005; 23:861-865.

17. Nakamura T, Sugaya $\mathrm{T}$, Kawagoe $\mathrm{Y}$, et al. Azelnidipine reduces urinary protein excretion and urinary liver-type fatty acid binding protein in patients with hypertensive chronic kidney disease. Am J Med Sci 2007;333:321-326.

18. Kojima M, Okubo S, Mizubayashi R, et al. Kidney-protective effects of azelnidipine versus a diuretic in combination with olmesartan in hypertensive patients with diabetes and albuminuria: a randomized study. Nephrol Dial Transplant. 2013;28:1802-1810

19. Ogawa S, Matsushima M, Mori T, et al. Identification of the stages of diabetic nephropathy at which angiotensin II receptor blockers most effectively suppress albuminuria. Am J Hypertens. 2013; 26: 1054-1069.

20. Fujita $T$, Ando $K$, Nishimura $H$, et al. On behalf of the Cilnidipine versus Amlodipine Randomized Trial for Evaluation in Renal Disease (CARTER) Study Investigators: Antiproteinuric effect of the calcium channel blocker cilnidipine added to renin-angiotensin inhibition in hypertensive patients with chronic renal disease. Kidney Int 2007; 72: 1543-1549. 